\title{
A Note on The Rogers-Fine Identity
}

\author{
Jian-Ping Fang* \\ Department of Mathematics, Huaiyin Teachers College, Huaian, Jiangsu 223300, P. R. China \\ Department of Mathematics, East China Normal University, Shanghai 200062, P. R. China \\ fjp7402@163.com
}

Submitted: May 29, 2006; Accepted: Jul 30, 2007; Published: Aug 9, 2007

Mathematics Subject Classifications: 05A30; 33D15; 33D60; 33D05

\begin{abstract}
In this paper, we derive an interesting identity from the Rogers-Fine identity by applying the $q$-exponential operator method.
\end{abstract}

\section{Introduction and main result}

Following Gasper and Rahman [7], we write

$$
\begin{gathered}
(a ; q)_{0}=1, \quad(a ; q)_{n}=(1-a)(1-a q) \cdots\left(1-a q^{n-1}\right), n=1, \cdots, \infty, \\
{ }_{r} \Phi_{s}\left(\begin{array}{c}
a_{1}, \cdots, a_{r} \\
b_{1}, \cdots, b_{s}
\end{array} ; q, x\right)=\sum_{n=0}^{\infty} \frac{\left(a_{1}, a_{2}, \cdots, a_{r} ; q\right)_{n}}{\left(q, b_{1}, \cdots, b_{s} ; q\right)_{n}}\left[(-1)^{n} q^{n(n-1) / 2}\right]^{1+s-r} x^{n} .
\end{gathered}
$$

For convenience, we take $|q|<1$ in this paper.

Recall that the Rogers-Fine identity $[1,2,6,10]$ is expressed as follows:

$$
\sum_{n=0}^{\infty} \frac{(\alpha ; q)_{n}}{(\beta ; q)_{n}} \tau^{n}=\sum_{n=0}^{\infty} \frac{(\alpha ; q)_{n}(q \alpha \tau / \beta ; q)_{n}\left(1-\alpha \tau q^{2 n}\right)}{(\beta ; q)_{n}(\tau ; q)_{n+1}}(\beta \tau)^{n} q^{n^{2}-n}
$$

This identity (1) is one of the fundamental formulas in the theory of the basic hypergeometric series. In this paper, we derive an interesting identity from (1) by applying the $q$-exponential operator method. As application, we give an extension of the terminating very-well-poised ${ }_{6} \Phi_{5}$ summation formula. The main result of this paper is:

* Jian-Ping Fang supported by Doctorial Program of ME of China 20060269011. 
Theorem 1.1. Let $a_{-1}, a_{0}, a_{1}, a_{2}, \cdots, a_{2 t+2}$ be complex numbers, $\left|a_{2 i}\right|<1$ with $i=$ $0,1,2, \cdots, t+1$, then for any non-negative integer $M$, we have

$$
\begin{aligned}
\sum_{n=0}^{M} & \frac{\left(q^{-M}, c, a_{2}, a_{4}, \cdots, a_{2 t+2} ; q\right)_{n}}{\left(\beta, b, a_{1}, a_{3}, \cdots, a_{2 t+1} ; q\right)_{n}} \tau^{n} \\
= & \sum_{m=0}^{M} \frac{\left(q^{-M} ; q\right)_{m}\left(\tau q^{1-M} / \beta ; q\right)_{m}\left(1-\tau q^{2 m-M}\right)}{(\beta ; q)_{m}(\tau ; q)_{m+1}}(\beta \tau)^{m} q^{m^{2}-m} \\
& \times \prod_{j=0}^{t+1} \frac{\left(a_{2 j} ; q\right)_{m}}{\left(a_{2 j-1} ; q\right)_{m}} \sum_{m_{1}=0}^{m} \frac{\left(q^{-m}, q^{1-m} / \beta, b / c ; q\right)_{m_{1}}}{\left(q, q^{1-M} \tau / \beta, q^{1-m} / c ; q\right)_{m_{1}}} \quad \sum_{0 \leq m_{t+2} \leq m_{t+1} \leq \cdots \leq m_{2} \leq m_{1}} \\
& \quad \prod_{i=1}^{t+1} \frac{\left(q^{-m_{i}}, q^{1-m} / a_{2 i-3}, a_{2 i-1} / a_{2 i} ; q\right)_{m_{i+1}}}{\left(q, q^{1-m} / a_{2 i}, q^{1-m_{i}} a_{2 i-2} / a_{2 i-3} ; q\right)_{m_{i+1}}} q^{m_{1}+m_{2}+\cdots+m_{t+2}},
\end{aligned}
$$

where $t=-1,0,1,2, \cdots, \infty, c=a_{0}$ and $b=a_{-1}$.

\section{The proof of the theorem and its application}

Before our proof, let's first make some preparations. The $q$-differential operator $D_{q}$ and $q$-shifted operator $\eta$ (see $[3,4,8,9]$ ), acting on the variable $a$, are defined by:

$$
D_{q}\{f(a)\}=\frac{f(a)-f(a q)}{a} \text { and } \eta\{f(a)\}=f(a q) .
$$

Rogers [9] first used them to construct the following q-operator

$$
E(d \theta)=(-d \theta ; q)_{\infty}=\sum_{n=0}^{\infty} \frac{q^{(n-1) n / 2}(d \theta)^{n}}{(q ; q)_{n}}
$$

where $\theta=\eta^{-1} D_{q}$. Note that, Rogers used the symbol $q \delta$ to denote $\theta$ [9]. Then he applied it to derive relationships between special functions involving certain fundamental $q$-symmetric polynomials. This operator theory was developed by Chen and Liu [4] and Liu [8]. They employed (3) to obtain many classical $q$-series formulas. Later Bowman [3] studied further results of this operator and gave convergence criteria. He used it to obtain results involving $q$-symmetric expansions and $q$-orthogonal polynomials. Inspired by their work, we constructed the following $q$-exponential operator [5]

Definition 2.1. Let $\theta=\eta^{-1} D_{q}, b, c$ are complex numbers. We define

$$
{ }_{1} \Phi_{0}\left(\begin{array}{c}
b \\
-
\end{array} q,-c \theta\right)=\sum_{n=0}^{\infty} \frac{(b ; q)_{n}(-c \theta)^{n}}{(q ; q)_{n}} .
$$

In [5], we have applied it to obtain some formal extensions of $q$-series formulas. Notice that the operator $E(d \theta)$ follows (4) by setting $c=d h, b=1 / h$, and taking $h=0$. The following operator identities were given in [5]: 
Lemma 2.1. If $s / \omega=q^{-N},|c s t / \omega|<1$, where $N$ is a non-negative integer, then

$$
\begin{aligned}
& { }_{1} \Phi_{0}\left(\begin{array}{c}
b \\
-
\end{array} q,-c \theta\right)\left\{\frac{(a s, a t ; q)_{\infty}}{(a \omega ; q)_{\infty}}\right\} \\
& =\frac{(a s, a t, b c t ; q)_{\infty}}{(a \omega, c t ; q)_{\infty}} \Phi_{2}\left(\begin{array}{ccc}
b, & s / \omega, & q / a t \\
& q / c t, & q / a \omega
\end{array} ; q, q\right) .
\end{aligned}
$$

Lemma 2.2. For $|c s|<1$,

$$
{ }_{1} \Phi_{0}\left(\begin{array}{c}
b \\
-
\end{array} ;,-c \theta\right)\left\{(a s ; q)_{\infty}\right\}=\frac{(a s, b c s ; q)_{\infty}}{(c s ; q)_{\infty}} .
$$

Now let's return to the proof of Theorem 1.1. Employing

$$
(q / a ; q)_{n}=(-a)^{-n} q^{n(n+1) / 2} \frac{\left(q^{-n} a ; q\right)_{\infty}}{(a ; q)_{\infty}}
$$

and setting $\alpha=q^{-M}$ in (1), we rewrite the new expression as follows:

$$
\begin{aligned}
\sum_{n=0}^{M}\left(q^{-M} ; q\right)_{n}\left(\beta q^{n} ; q\right)_{\infty} \tau^{n}= & \sum_{n=0}^{M} \frac{\left(q^{-M} ; q\right)_{n}\left(1-q^{2 n-M} \tau\right)}{(\tau ; q)_{n+1}}\left(-q^{-M} \tau^{2}\right)^{n} \\
& \times q^{n(3 n-1) / 2} \frac{\left(\beta q^{M-n} / \tau, \beta q^{n} ; q\right)_{\infty}}{\left(\beta q^{M} / \tau ; q\right)_{\infty}}
\end{aligned}
$$

Applying the operator ${ }_{1} \Phi_{0}\left(\begin{array}{c}b \\ -\end{array} ; q,-c \theta\right)$ to both sides of $(8)$ with respect to the variable $\beta$ then we have

$$
\begin{aligned}
\sum_{n=0}^{M} & \left(q^{-M} ; q\right)_{n} \tau_{1}^{n} \Phi_{0}\left(\begin{array}{c}
b \\
-
\end{array} ; q,-c \theta\right)\left\{\left(\beta q^{n} ; q\right)_{\infty}\right\} \\
= & \sum_{n=0}^{M} \frac{\left(q^{-M} ; q\right)_{n}\left(1-\tau q^{2 n-M}\right)}{(\tau ; q)_{n+1}}\left(-q^{-M} \tau^{2}\right)^{n} \\
& \times q^{n(3 n-1) / 2}{ }_{1} \Phi_{0}\left(\begin{array}{c}
b \\
-
\end{array} ;,-c \theta\right)\left\{\frac{\left(\beta q^{M-n} / \tau, \beta q^{n} ; q\right)_{\infty}}{\left(q^{M} \beta / \tau ; q\right)_{\infty}}\right\} .
\end{aligned}
$$

By (5) and (6), we have the relation

$$
\begin{aligned}
\sum_{n=0}^{M} \frac{\left(q^{-M}, c ; q\right)_{n}}{(\beta, b c ; q)_{n}} \tau^{n}= & \sum_{n=0}^{M} \frac{\left(q^{-M} ; q\right)_{n}\left(q^{1-M} \tau / \beta, c ; q\right)_{n}\left(1-\tau q^{2 n-M}\right)}{(\beta, b c ; q)_{n}(\tau ; q)_{n+1}}(\beta \tau)^{n} q^{n^{2}-n} \\
& \times{ }_{3} \Phi_{2}\left(\begin{array}{c}
q^{-n}, b, q^{1-n} / \beta \\
q^{1-M} \tau / \beta, q^{1-n} / c
\end{array} ; q, q\right) .
\end{aligned}
$$


Using (7) again, we rewrite (9) as follows:

$$
\begin{aligned}
\sum_{n=0}^{M} \frac{\left(q^{-M}, c ; q\right)_{n}}{(\beta ; q)_{n}} \tau^{n}\left(b c q^{n} ; q\right)_{\infty}= & \sum_{n=0}^{M} \frac{\left(q^{-M} ; q\right)_{n}\left(q^{1-M} \tau / \beta, c ; q\right)_{n}\left(1-\tau q^{2 n-M}\right)}{(\beta ; q)_{n}(\tau ; q)_{n+1}}(\beta \tau)^{n} q^{n^{2}-n} \\
& \times \sum_{n_{1}=0}^{n} \frac{\left(q^{-n}, q^{1-n} / \beta ; q\right)_{n_{1}} q^{n_{1}}}{\left(q, q^{1-M} \tau / \beta, q^{1-n} / c ; q\right)_{n_{1}}} \frac{\left(b c q^{n} ; q\right)_{\infty}}{\left(b q^{n_{1}} ; q\right)_{\infty}} .
\end{aligned}
$$

Applying the operator ${ }_{1} \Phi_{0}\left(\begin{array}{c}a_{1} \\ -\end{array} ; q,-a_{2} \theta\right)$ to both sides of (10) with respect to the variable $b$, from (5) and (6) and simplifying then we have

$$
\begin{aligned}
& \sum_{n=0}^{M} \frac{\left(q^{-M}, c, a_{2} c ; q\right)_{n}}{\left(\beta, b c, a_{1} a_{2} c ; q\right)_{n}} \tau^{n}=\sum_{n=0}^{M} \frac{\left(q^{-M} ; q\right)_{n}\left(q^{1-M} \tau / \beta, c, a_{2} c ; q\right)_{n}\left(1-\tau q^{2 n-M}\right)}{\left(\beta, b c, a_{1} a_{2} c ; q\right)_{n}(\tau ; q)_{n+1}}(\beta \tau)^{n} q^{n^{2}-n} \\
& \times \sum_{n_{1}=0}^{n} \frac{\left(q^{-n}, q^{1-n} / \beta, b ; q\right)_{n_{1}}}{\left(q, q^{1-M} \tau / \beta, q^{1-n} / c ; q\right)_{n_{1}}} q^{n_{1}} \sum_{n_{2}=0}^{n_{1}} \frac{\left(q^{-n_{1}}, q^{1-n} / b c, a_{1} ; q\right)_{n_{2}}}{\left(q, q^{1-n} / a_{2} c, q^{1-n_{1}} / b ; q\right)_{n_{2}}} q^{n_{2}} .
\end{aligned}
$$

Replacing $b c$ by $b$ in (9), we have the case of $t=-1$. If we replace $\left(b c, a_{2} c, a_{1} a_{2} c\right)$ by $\left(b, a_{2}, a_{1}\right)$ in (11) respectively, we obtain the case of $t=0$.

By induction, similar proof can be performed to get the equation (2).

Letting $t=-1$ in (2), and then setting $b=q^{1-M} \tau / \beta$, we have the following identity:

Corollary 2.1. If $|c|<1$, then

$$
\begin{aligned}
& \sum_{n=0}^{M} \frac{\left(q^{-M}, c ; q\right)_{n}}{\left(\beta, q^{1-M} c \tau / \beta ; q\right)_{n}} \tau^{n} \\
& =\sum_{n=0}^{M} \frac{\left(q^{-M} ; q\right)_{n}\left(q^{1-M} \tau / \beta, \beta / c ; q\right)_{n}\left(1-\tau q^{2 n-M}\right)}{\left(\beta, q^{1-M} c \tau / \beta ; q\right)_{n}(\tau ; q)_{n+1}}(-c \tau)^{n} q^{n(n-1) / 2} .
\end{aligned}
$$

Combined with (12), we can get the following extension of the terminating very-wellpoised ${ }_{6} \Phi_{5}$ summation formula:

Theorem 2.1. For $|c|<1,|e|<1$ and $|\tau|<1$

$$
\begin{aligned}
& \sum_{n=0}^{M} \frac{\left(1-\tau q^{2 n}\right)\left(\tau, q^{-M} ; q\right)_{n}}{(1-\tau)\left(q, \tau q^{M+1} ; q\right)_{n}}\left(-c \tau q^{M}\right)^{n} q^{n(n-1)^{2}} \frac{(q / c, e \tau ; q)_{n}}{(c \tau, d e \tau ; q)_{n}} \\
& \times{ }_{3} \Phi_{2}\left(\begin{array}{c}
q^{-n}, \\
q^{1-n} / c \tau, \\
q^{1-n} / e \tau, \quad q / c
\end{array} ; q, q\right)=\frac{(\tau q, e \tau ; q)_{M}}{(c \tau, d e \tau ; q)_{M}} .
\end{aligned}
$$

Proof. Setting $\beta=q$ and replacing $\tau$ by $\tau q^{M}$ in (12), we have

$$
\frac{(\tau q ; q)_{M}}{(c \tau ; q)_{M}}=\sum_{n=0}^{M} \frac{\left(1-\tau q^{2 n}\right)\left(\tau, q / c, q^{-M} ; q\right)_{n}}{(1-\tau)\left(q, c \tau, \tau q^{M+1} ; q\right)_{n}}\left(-c \tau q^{M}\right)^{n} q^{n(n-1) / 2} .
$$


Employing (7), we rewrite (14) as follows:

$$
(\tau q ; q)_{M}\left(c \tau q^{M} ; q\right)_{\infty}=\sum_{n=0}^{M} \frac{\left(1-\tau q^{2 n}\right)\left(\tau, q^{-M} ; q\right)_{n}}{(1-\tau)\left(q, \tau q^{M+1} ; q\right)_{n}}\left(\tau q^{M}\right)^{n} q^{n^{2}} \frac{\left(c q^{-n}, c \tau q^{n} ; q\right)_{\infty}}{(c ; q)_{\infty}}
$$

Applying the operator ${ }_{1} \Phi_{0}\left(\begin{array}{c}d \\ -\end{array} ; q,-e \theta\right)$ to both sides of $(15)$ with respect to the variable $c$, using (5) and (6) and simplifying then we complete the proof.

Taking $d=q / c$ then setting $e=c f / q$ in (13), we have

Corollary 2.2 (The terminating very-well-poised ${ }_{6} \Phi_{5}$ summation formula).

$$
{ }_{6} \Phi_{5}\left(\begin{array}{c}
q^{-M}, \tau, q \sqrt{\tau},-q \sqrt{\tau}, q / c, q / f \\
\tau q^{M+1}, \sqrt{\tau},-\sqrt{\tau}, c \tau, f \tau
\end{array} ; q, c f \tau q^{M-1}\right)=\frac{(\tau q, c f \tau / q ; q)_{M}}{(c \tau, f \tau ; q)_{M}} .
$$

Remark: In the context of this paper, convergence of the basic hypergeometric series is no issue at all because they are terminating $q$-series.

Acknowledgements: I would like to thank the referees for their many valuable comments and suggestions. And I am grateful to professor D. Bowman who presented me some information about reference [3].

\section{References}

[1] G. E. Andrews, A Fine Dream, Int. J. Number Theory, In Press, 2006.

[2] B. C. Berndt, Ae Ja Yee, Combinatorial Proofs of Identities in Ramanujan's Lost Notebook Associated with the Rogers-Fine Identity and False Theta Functions, Ann. Comb., 7 (2003), 409-423.

[3] D. Bowman, q-Differential Operators, Orthogonal Polynomials, and Symmetric Expansions, Mem. Amer. Math. Soc., 159 (2002).

[4] W. Y. C. Chen, Z.-G. Liu, Parameter Augmentation For Basic Hypergeometric Series I, In: B. E. Sagan, R. P. Stanley (Eds.), Mathematical Essays in Honor of GianCarlo Rota, Progr. Math., 161 (1998), 111-129.

[5] J.-P. Fang, q-Differential operator identities and applications, J. Math. Anal. Appl., 332 (2007), 1393-1407.

[6] N. J. Fine, Basic Hypergeometric Series and Applications, American Mathematical Society, Providence, RI, 1988.

[7] G. Gasper, M. Rahman, Basic Hypergeometric Series, Cambridge University Press, Cambridge, Ma, 1990.

[8] Z.-G. Liu, Some Operator Identities and q-Series Transformation Formulas, Discrete Math., 265 (2003), 119-139.

[9] L. J. Rogers, On the expansion of some infinite products, Proc. London Math.Soc., 24 (1893), 337-352.

[10] L. J. Rogers, On two theorems of combinatory analysis and some allied identities, Proc. London Math.Soc., 16 (1917), 315-336. 